\title{
Pioglitazone, a Peroxisome Proliferator- Activated Receptor $y$ Agonist, Ameliorates Chronic Kidney Disease by Enhancing Antioxidative Capacity and Attenuating Angiogenesis in the Kidney of a 5/6 Nephrectomized Rat Model
}

\author{
Li Sun ${ }^{\mathrm{a}}$ Quan Yuan ${ }^{\mathrm{b}}$ Tianhua Xua Li Yao Jiangmin Feng ${ }^{\mathrm{a}} \quad$ Jianfei Ma ${ }^{\mathrm{a}}$ Lining \\ Wang $^{a}$ Changlong Luc Danan Wang ${ }^{c}$ \\ aDepartment of Nephrology, The First Affiliated Hospital of China Medical University, Shenyang, \\ ${ }^{b}$ Department of Orthopedics, Shengjing Hospital of China Medical University, Shenyang, 'Department \\ of Immunology, China Medical University, Shenyang, People's Republic of China
}

\section{Key Words}

Peroxisome proliferator-activated receptor $\gamma$ • Pioglitazone $\cdot 5 / 6$ nephrectomy $\cdot$ Hypoxiainducible factor 1 alpha $\bullet$ Vascular endothelial growth factor

\begin{abstract}
Background/Aims: Pioglitazone is a type of peroxisome proliferator-activated receptor $\gamma$ agonist and is capable of alleviating renal ischemia-reperfusion injury. Methods: A5/6 nephrectomized rat model was established to induce renal impairments mimicking chronic kidney diseases (CKDs). The effect of pioglitazone on renal structure, function, antioxidative capacity, and angiogenesis in the nephrectomized rats was assessed. Moreover, the expression of HIF-1 $\alpha$, eNOS, VEGF, Flt-1 and Flk-1 was determined to reveal the possible pathways through which pioglitazone exerted its beneficial effect on CKDs. Results: Subtotal nephrectomy caused severe damages to rat renal tissues, and administration of pioglitazone dramatically restored the structure and function of the kidney, which was evidenced by Periodic acidSchiff staining and the reduced levels of urinary proteins, blood urea nitrogen, and creatinine. Furthermore, pioglitazone decreased the level of malondialdehyde and increased the level of superoxide dismutase in the injured renal tissues, suggesting that the antioxidative capacity in the injured kidney was augmented by pioglitazone. Additionally, pioglitazone inhibited HIF- $1 \alpha$-dependent angiogenesis by down-regulating the expression of a panel of angiogenic factors. Conclusion: The current study demonstrates that pioglitazone benefits renal failure through activation of the antioxidative system and inhibition of angiogenesis in the injured kidney. Our study provides preliminary evidences for the potential application of this agent in the treatment of CKDs.




\section{Introduction}

Chronic kidney disease (CKD) is characterized by relentlessly progressive scarring of the renal parenchyma, and it causes a large number of mortality and morbidity worldwide. Persistent exposure to CKD will lead to end-stage renal disease (ESRD) and has casted a severe threat to the public health [1]. Recent studies regarding the pathology of CKD and ESRD emphasize the correlation between impaired renal function and the degree of tubulointerstitial damage [2-5]. This finding further leads to a broad recognition that the final common pathway of renal failure may take effect principally in the tubulointerstitium [6-8]. Moreover, based on the chronic hypoxia hypothesis by Fine et al. [9], which places chronic hypoxia in the center of tubulointerstitial injury, increasing investigations regarding the role of chronic hypoxia and tubulointerstitial injury in CKD have been conducted. The findings substantiate the potential of controlling chronic hypoxia and angiogenesis as an effective therapy for CKD [10-12].

Peroxisome proliferator-activated receptor $\gamma$ (PPAR $\gamma$ ) is a member of the nuclear hormone receptor superfamily, members of which are ligand-activated transcription factors related to retinoid, steroid and thyroid hormone receptors [13]. By binding to the specific PPAR response elements (PPREs) in the promoter region, PPARs, regulate the expression of target genes as heterodimers with retinoid X receptors (RXRs) [14]. In the recent years, a physiological function of PPAR $\gamma$ as a major factor in the differentiation of adipocytes has been revealed [15]. In addition, selective activation of PPAR $\gamma$ with specific agonists has been shown to exert therapeutic effects on cardiovascular disease, diabetes, inflammation and tumors [16-20]. In the case of kidney disorders, pioglitazone, a PPAR $\gamma$ agonist, is capable of protecting the kidney from ischemia-reperfusion injury by enhancing the antioxidant capacity [21]. Moreover, pioglitazone can ameliorate endothelial dysfunction and increase the expression of vascular endothelial growth factor (VEGF), which contributes to angiogenesis and improvement of metabolic syndrome-induced renal injury [22-24]. However, there exists contradiction on the function of pioglitazone on the antioxidative machinery and angiogenesis. In a study of diabetic nephropathy, Dromparis et al. demonstrated that pioglitazone dramatically reduced the expression of hypoxia-inducible factor 1 alpha (HIF-1 $\alpha$ ) and VEGF [25]. Therefore, the effect of pioglitazone on hypoxia- or capillary loss-induced renal injury might depend on the specific disease condition and the time of drug administration. Thus, exploration of the underlying mechanism for pioglitazonerelieved renal damage will provide a theoretical foundation for the potential application of pioglitazone in treating CKD.

In the present study, the effect of pioglitazone on renal injury was assessed in vivo with a 5/6 nephrectomized ( $\mathrm{Nx}$ ) rat model [26]. The direct effect of pioglitazone on the impaired structure and function of the kidney was determined by periodic acid-Schiff (PAS) staining and the measurements of proteinuria, blood urea nitrogen (BUN) and creatinine (Cr). Furthermore, the impact of pioglitazone on the expression of anti-oxidative and angiogenic factors were detected in order to reveal the possible mechanism underlying pioglitazonemediated alleviation of CKD symptoms.

\section{Materials and Methods}

Chemicals and animals

Pioglitazone was purchased from Meilune (Catalog No. MB1185, Dalian, China). PPAR pathway inhibitor GW9662 was obtained from Sigma (Catalog No. N6191, USA). Antibodies against HIF-1 $\alpha$, Flt1, and Flk-1 were purchased from Santa Cruz (USA). Antibodies against CD31, $\alpha$ SMA, eNOS, and VEGF were purchased from Boster (China). Adult male Sprague Dawley (SD) rats (weighting 200-220 g) were provided by the Experimental Animal Center of China Medical University. The animals were housed in cages at $20-25^{\circ} \mathrm{C}$ with a constant humidity $(55 \pm 5 \%)$, and provided with water and food ad libitum. All the animal experiments were performed in accordance with the Animal Care Guidelines for the Care and Use 


\section{Cellular Physiology Cell Physiol Biochem 2016;38:1831-1840 \\ \begin{tabular}{l|l} 
and Biochemistry Published online: May 09, 2016 & $\begin{array}{l}\text { C } 2016 \text { The Author(s). Published by S. Karger AG, Basel } \\
\text { www.karger.com/cpb }\end{array}$ \\
\hline
\end{tabular}}

Sun et al.: Pioglitazone Antagonizes CKD via Augmentation of Antioxidative Capacity and Block of Angiogenesis

of Laboratory Animals and the protocol approved by the Institutional Animal Ethics Committee of China Medical University.

Establishment of the rat model with chronic renal failure

For induction of chronic renal failure by $5 / 6$ nephrectomy, the rats were anesthetized with pentobarbital sodium $(50 \mathrm{mg} / \mathrm{kg}$ ). A left flank incision was made to expose the left kidney. Then the renal artery was temporarily occluded, and the upper and the lower third of the kidney was ligated and excised. After suture of the incision with polypropylene suture, the rats were returned to the cages for recovery for one week. Thereafter, a right flank incision was made and the entire right kidney was removed. After recovery for one more week, the rats were given different treatments as described below. 24 SD rats were randomly divided into four groups (six in each group). (A) sham group: the rats underwent all the procedures of 5/6 nephrectomy except for the removal of kidney; (B) Nx group: the rats underwent 5/6 nephrectomy and were gavaged with the vehicle of pioglitazone every day for 8 weeks; (C) Nx+pioglitazone group, the Nx rats were gavaged with $10 \mathrm{mg} / \mathrm{kg}$ pioglitazone every day for 8 weeks; (D) Nx+pioglitazone+GW9662 group: $1 \mathrm{~h}$ prior to pioglitazone administration, the rats were intraperitoneally injected with $1 \mathrm{mg} / \mathrm{kg}$ GW9662. After 8-week treatment, all the experimental animals were sacrificed. The kidneys and the serum were collected for the subsequent experiments.

Periodic acid-Schiff (PAS) staining and renal function assay

The injuries in the glomerulus and renal tubules were examined using PAS staining. Briefly, the kidneys were fixed in $10 \%$ buffered formalin. The sample was cut into 2 - $\mu$ m sections, stained with periodic acid-Schiff, and scored as described previously [27]. The sections were examined by microscopy at $400 \times$ magnification. To assess the effect of pioglitazone on renal function, the content of proteinuria within $24 \mathrm{~h}$, and the levels of BUN and Cr were measured using the specific kits (Catalog No. C013-1, C011-1, and A028-1, Nanjing Jiancheng Bioengineering Institute, China) according to the manufacturer's instructions.

\section{Detection of MDA, SOD, and NO}

To determine the effect of pioglitazone on the oxidative stress response initiated by renal failure, the levels of malondialdehyde (MDA) and superoxide dismutase (SOD) in the renal tissues were measured using the respective kits (Catalog No. A003-1 and A001-3, Nanjing Jiancheng Bioengineering Institute) according to the manufacturer's instructions. Total production of NO was also determined using the NO detection kit (Catalog No. S0023, Beyotime Biotechnology, China) according to the manufacturer's instruction.

Enzyme-linked immunosorbent assay (ELISA)

The contents of VEGF and Flt-1 in the serum samples were measured using the commercially available ELISA kits (Catalog No. EK0540, Boster, and Catalog No. DRE01252, WHB, China) according to the manufacturers' instructions.

\section{Immunohistochemical staining}

The expression levels of HIF- $1 \alpha$, VEGF, CD31, and $\alpha$ SMA in the renal tissues were detected by immunohistochemistry (IHC). The tissue sections were hydrated in serial concentrations of alcohol as follows: $70 \%$ for $2 \mathrm{~h}, 80 \%$ overnight, $90 \%$ for $2 \mathrm{~h}, 100 \%$ for 1 , and $100 \%$ for $1 \mathrm{~h}$. Afterwards, the sections were placed in dimethylbenzene and paraffin for $30 \mathrm{~min}$ before transferring into a $60^{\circ} \mathrm{C}$ incubator to form paraffin sections. The sections were then fixed in methanol solution with $3 \% \mathrm{H}_{2} \mathrm{O}_{2}$ for 15 min at room temperature, and washed with PBS for three times with 5 min each time. The sections were incubated with primary antibodies $(1: 100)$ against different proteins of interest at $4^{\circ} \mathrm{C}$ overnight. After washing with 0.01 M PBS for $4 \times 5 \mathrm{~min}$, the sections were incubated with HRP labeled secondary antibodies $(1: 200)$ at $37^{\circ} \mathrm{C}$ for $30 \mathrm{~min}$, followed by washing with PBS. DAB was added to the sections for 3-10 min reaction, which was stopped by $\mathrm{ddH}_{2} \mathrm{O}$. The sections were re-stained with haematoxylin and dehydrated. IHC results were assessed by scanning the sections using Aperio ScanScope GL (Aperio Technologies, Vista, CA, USA) at 400× magnification.

Reverse transcription-quantitative PCR (RT-qPCR)

The effect of pioglitazone on the expression of angiogenic factors in the Nx rats was further determined by RT-qPCR. Total RNAs in the kidney samples were extracted using a RNA extraction kit according to the 
manufacturer's instructions (Catalog No. RP1201, BioTeke, China). $\beta$-actin was selected as the reference gene. cDNA templates were obtained by reverse transcription of the RNAs using Super M-MLV reverse transcriptase (Catalog No. PR6502, BioTeke). The final quantitative PCR reaction mixture of $20 \mu \mathrm{L}$ volume contained $10 \mu \mathrm{L}$ SYBR GREEN mastermix, $0.5 \mu \mathrm{L}$ of each primers of a gene (HIF-1 $\alpha$, forward: 5'- CTC CCA TAC AAG GCA GCA GAAAC-3', reverse: 5'-AGA AAC GAA ACC CCA CAG ACAAC-3'; eNOS, forward: 5'-CAG GCA TCA CCA GGA AGAA-3', reverse: 5'-TCA GAG CCA TAC AGG ATA GTCG-3'; VEGF, forward: 5'- ATG AAC TTT CTG CTG TCT TGG-3', reverse: 5'- TCA CCG CCT CGG CTT GTC ACA-3'; Flt-1, forward: 5'- CCG TGT ATG GCA TCC CTCA-3', reverse: 5'-CCA CCA CCA ATG TGC TAACC-3'; Flk-1, forward: 5'-TTT TGA GCA CCT TGA CCATA-3', reverse: 5'-TTC ACA GGG ATT CGG ACTT-3'; $\beta$-actin forward: 5'-GGA GAT TAC TGC CCT GGC TCC TAGC-3', reverse: 5'-GGC CGG ACT CAT CGT ACT CCT GCTT-3'), $1 \mu \mathrm{L}$ cDNA template, and $8 \mu \mathrm{L}$ RNase free $\mathrm{H}_{2} \mathrm{O}$. Thermal cycling parameters for the amplification were set as follows: a denaturation step at $95^{\circ} \mathrm{C}$ for $10 \mathrm{~min}$, followed by 40 cycles of $95^{\circ} \mathrm{C}$ for $10 \mathrm{~s}, 60^{\circ} \mathrm{C}$ for $20 \mathrm{~s}$ and $72^{\circ} \mathrm{C}$ for $30 \mathrm{~s}$. Relative expression levels of the target genes were calculated with the Data Assist Software version 3.0 (Applied Biosystems/Life Technologies) for the values of $2^{-\Delta \Delta c t}$.

\section{Western blotting assay}

The proteins were extracted using the Total Protein Extraction Kit according to the manufacturer's instructions (Catalog No. WLA019, Wanleibio, China). $\beta$-actin was used as the internal reference protein. Protein concentration was determined using the BCA method. $40 \mu \mathrm{g}$ protein in $20 \mu \mathrm{L}$ volume was subjected to $10 \%$ sodium dodecylsulfate polyacrylamide gel electrophoresis (SDS-PAGE). After transferring the proteins onto polyvinylidene difluoride (PVDF) membranes, the membranes were washed with TTBS for 5 min and then incubated with skim milk powder solution for $1 \mathrm{~h}$. Primary antibody against HIF-1 $\alpha$ (1:200), eNOS (1:400), VEGF (1:400), Flt-1 (1:200), Flk-1 (1:200) or $\beta$-actin (1:1000) was added and the membranes were incubated at $4^{\circ} \mathrm{C}$ overnight. Following washing with TTBS, the membranes were incubated with HRPconjugated IgG secondary antibodies $(1: 5000)$ for $45 \mathrm{~min}$ at $37^{\circ} \mathrm{C}$. After washing, the blots were developed using Beyo ECL Plus reagent and scanned in the Gel Imaging System. The relative expression levels of the target proteins were calculated with Gel-Pro-Analyzer (Media Cybernetics, USA).

Statistical analysis

All the data were expressed as mean $\pm S D$. One-way ANOVA and post-doc tests were performed using GraphPad Prism 6 (GraphPad Software, San Diego, CA) with a significance level of 0.05 .

\section{Results}

Administration of pioglitazone restored renal structure and function in Nx rats

The deteriorated structure of the kidney in the Nx rats was confirmed by PAS staining. Figure 1 shows the typical histological changes in each group. In the Nx rats, a dramatic increase in the sclerosed area was observed, whereas pioglitazone led to a decreased level of kidney sclerosis in the $\mathrm{Nx}$ rats compared with those without pioglitazone treatment. Moreover, GW9662 abolished the therapeutic effect of pioglitazone on the renal structure in the Nx rats, implying that pioglitazone-induced activation of PPAR $\gamma$ might play a critical in the restoration of renal structure.

Subtotal nephrectomy resulted in renal dysfunction, as evidenced by proteinuria and elevated levels of BUN and $\mathrm{Cr}$. The values of these three markers were significantly higher in the Nx group than that in the sham group $(P<0.05)$ (Fig. 2). Pioglitazone treatment effectively attenuated the elevation of proteinuria, BUN and $\mathrm{Cr}$ in the $\mathrm{Nx}$ rats $(P<0.05)$. Consistent with the results of PAS staining, GW9662 also inhibited the beneficial effect of pioglitazone on renal function.

Pioglitazone augmented anti-oxidative capacity in injured kidney

The production of MDA was significantly increased in the Nx rats as compared to the healthy rats in the sham group $(P<0.05)$ (Fig. 3A). The activity of SOD was also inhibited in the Nx group compared with the sham group (Fig. 3B). Compared with the Nx rats, 
Fig. 1. Histological examination of renal structure by PAS staining. Untreated $\mathrm{Nx}$ rat showed tubular atrophy, tubular dilation, hyperplasia and glomerulosclerosis, which were attenuated by pioglitazone treatment. Moreover, the therapeutic effect of pioglitazone was inhibited by the PPAR $\gamma$ inhibitor GW9662. Bar scale, $50 \mu \mathrm{m}$.
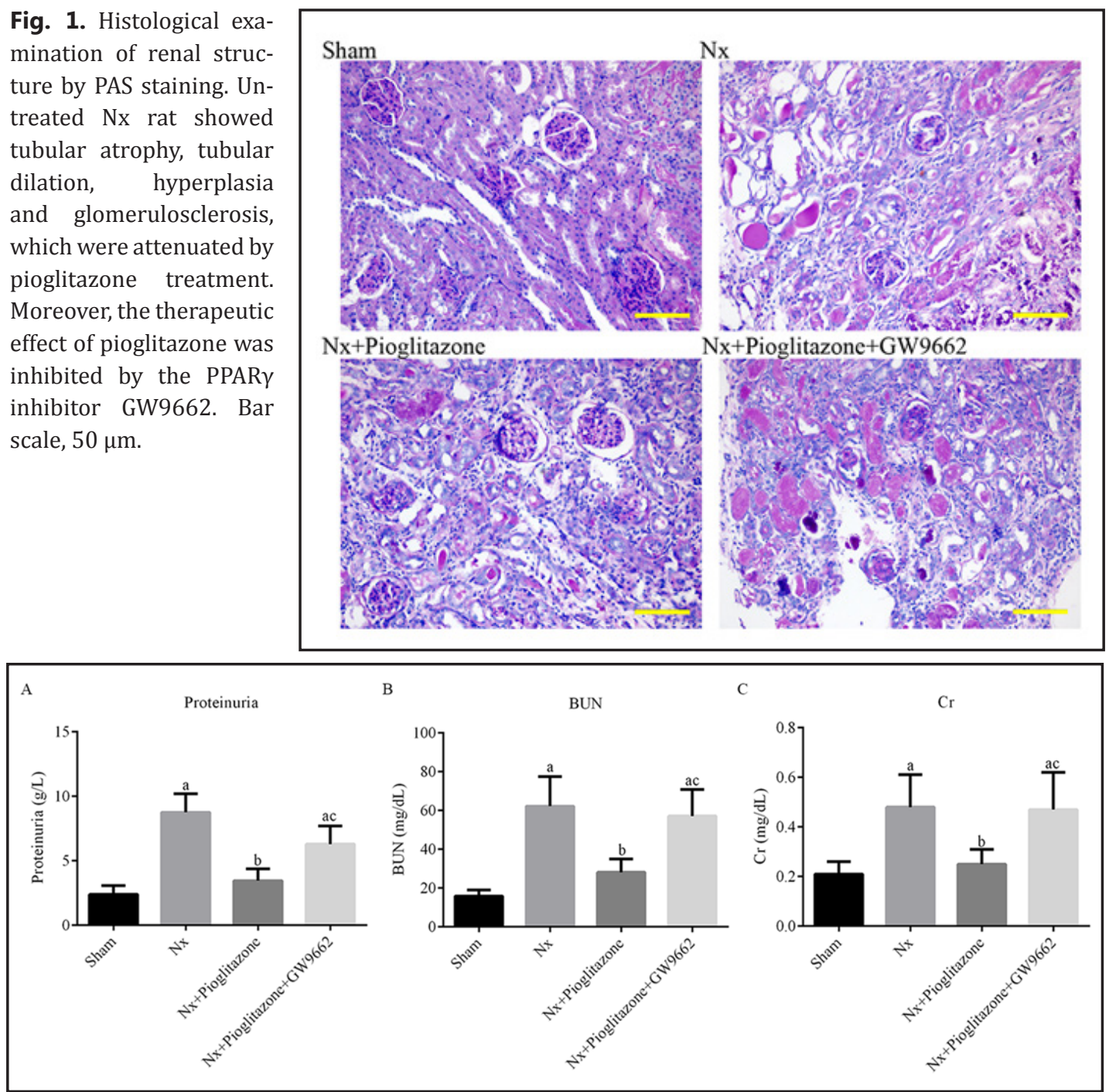

Fig. 2. Analysis of renal function. 5/6 nephrectomy induced elevation of proteinuria, BUN, and Cr, which were attenuated by administration of pioglitazone. The protective effect of pioglitazone against renal failure was inhibited by GW9662. Quantitative analysis of (A) proteinuria, (B) BUN, and (C) Cr. "a" indicates a significant difference from the sham group, $P<0.05$. "b" indicates a significant difference from the Nx group, $P<0.05$. "c" indicates a significant difference from the $\mathrm{Nx}+$ pioglitazone group, $P<0.05$.

pioglitazone treatment significantly reduced the level of MDA and increased the activity of SOD in the Nx kidney. By contrast, GW9662 almost abolished the effects of pioglitazone on MDA and SOD in the injured kidney.

\section{Pioglitazone inhibited angiogenesis in injured kidney}

The effect of pioglitazone on the angiogenesis process was assessed by a series of experiments. As shown in Fig. 4A, the production of NO was induced as a result of manipulated renal failure, and administration of pioglitazone decreased the level of NO to a normal level as in the sham group. Identical to the change pattern of NO, the serum level of VEGF was significantly elevated in the Nx rats as compared to the sham group $(P<0.05)$ (Fig. 4B), and it was decreased to normal by pioglitazone treatment. Similar results were observed for the level of Flt-1 in the serum (Fig. 4C).

The expression levels of HIF- $1 \alpha$, VEGF, CD31, and $\alpha$ SMA in the kidney were further analyzed by IHC. As shown in Fig. 5, the positively-stained cells were characterized by the 


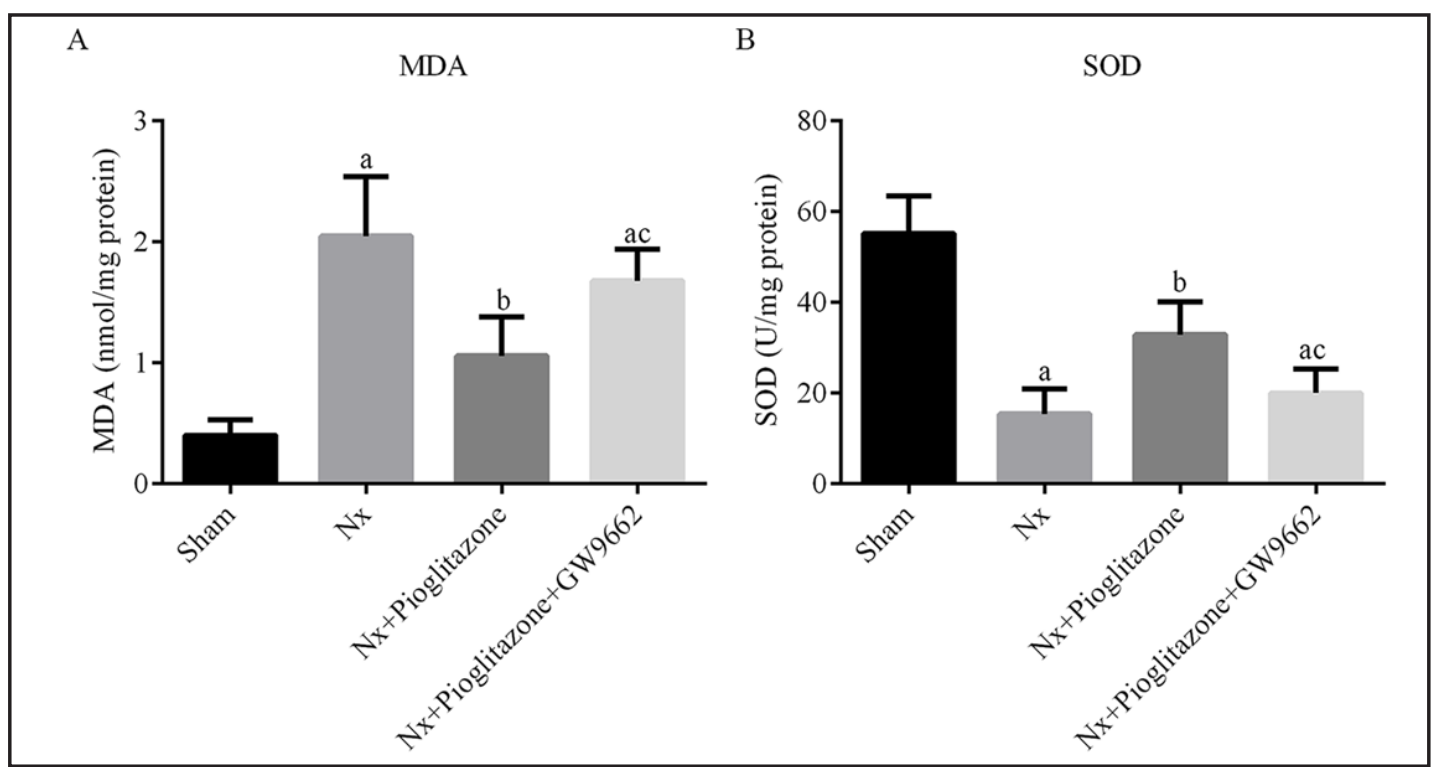

Fig. 3. The effect of pioglitazone on the anti-oxidative capacity in the injured kidney. The level of MDA was increased while the level of SOD was decreased as a result of 5/6 nephrectomy, indicating that the anti-oxidative capacity in the kidney was impaired. The levels of these two markers were restored after administration of pioglitazone, whereas the effect of pioglitazone on anti-oxidative response was inhibited by GW9662. (A) Quantitative analysis of MDA. (B) Quantitative analysis of SOD. "a" indicates a significant difference from the sham group, $P<0.05$. "b" indicates a significant difference from the Nx group, $P<0.05$. "c" indicates a significant difference from the $\mathrm{Nx}+$ pioglitazone group, $P<0.05$.

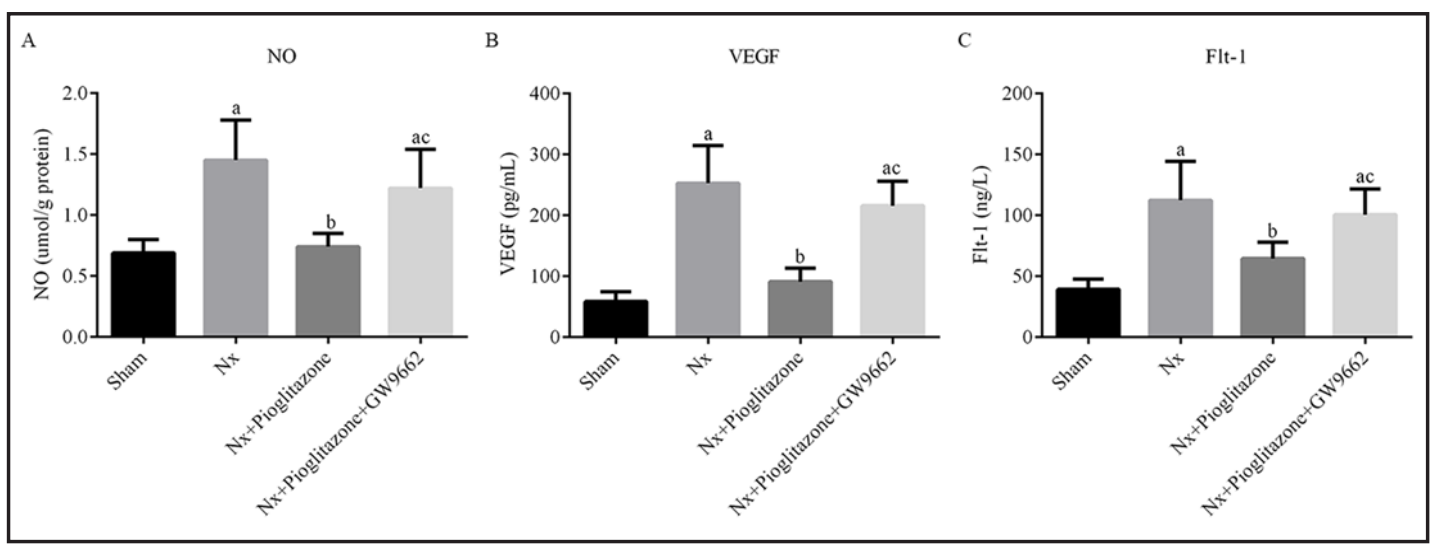

Fig. 4. The effect of pioglitazone on the serum levels of angiogenic molecules. The serum levels of NO, VEGF, Flt-1 were increased due to nephrectomy, and were reduced by pioglitazone. The effect of pioglitazone on the production of angiogenic molecules was inhibited by GW9662. This figure shows the quantitative analysis results of (A) NO, (B) VEGF and (C) Flt-1. "a" indicates a significant difference from the sham group, $P<0.05$. " $b$ " indicates a significant difference from the Nx group, $P<0.05$. "c" indicates a significant difference from the $\mathrm{Nx}+$ pioglitazone group, $P<0.05$.

presence of brownish-yellow particles. The expression levels of HIF-1 $\alpha$, VEGF, CD31, and $\alpha \mathrm{SMA}$ were all dramatically increased after induction of renal failure. In contrast, pioglitazone treatment markedly reduced the expression of these vascularization-related molecules. These results supported the conclusion that pioglitazone alleviates renal impairment through an anti-angiogenic manner.

To explore the possible pathways involved in pioglitazone-mediated renoprotection, the expression of HIF- $1 \alpha$, eNOS, VEGF, Flt-1 and Flk-1 were detected at both mRNA and 
Sun et al.: Pioglitazone Antagonizes CKD via Augmentation of Antioxidative Capacity and Block of Angiogenesis

Fig. 5. Representative images of IHC staining. The Nx rat showed up-regulated expression of HIF-1 $\alpha$, VEGF, CD31 and $\alpha$ SMA in the kidney, which were manifested by extensive distribution of brownish-yellow particles. Up-regulation of the angiogenic molecules was inhibited by pioglitazone, and such inhibitory effect of pioglitazone was eliminated by PPAR $\gamma$ inhibitor GW9662. (A) HIF- $1 \alpha$. (B) VEGF. (C) CD31. (D) $\alpha$ SMA. Bar scale, $50 \mu \mathrm{m}$.

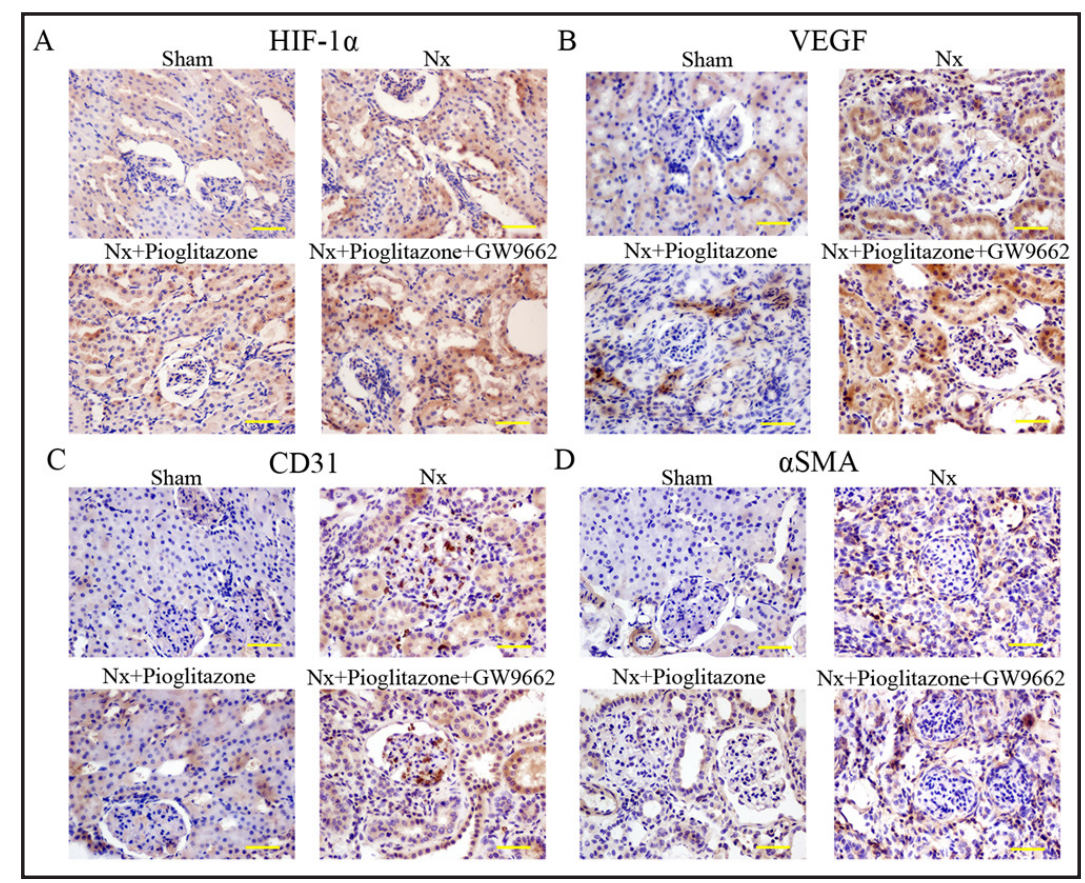

Fig. 6. The effect of pioglitazone on the expression of angiogenic molecules in the injured kidney. The expression of HIF- $1 \alpha$, eNOS, VEGF, Flt-1 and Flk-1 were up-regulated in the kidney tissues at both mRNA and protein levels in the $\mathrm{Nx}$ rats, and nephrectomy-induced up-regulation of these molecules was inhibited by pioglitazone. The effect of pioglitazone was abolished by PPAR $\gamma$ inhibitor GW9662. (A) Quantitative analysis of RT-qPCR results of five angiogenic factors. (B) Quantitative analysis and representative images of western blotting of the five factors. "a" indicates a significant difference from the sham group, $P<0.05$. "b" indicates a significant difference from the Nx group, $P<0.05$. "c" indicates a significant difference from the $\mathrm{Nx}+$ pioglitazone group, $P<0.05$.

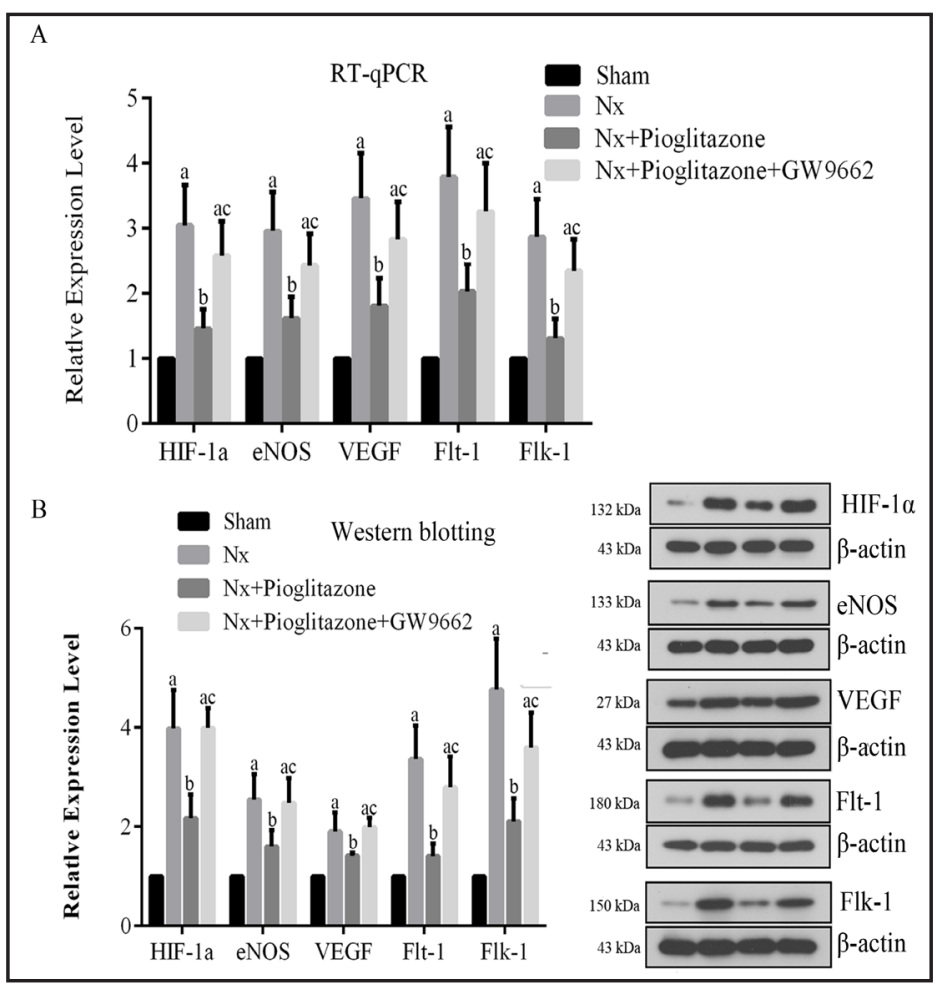

protein levels. As shown in Fig. 6, 5/6 nephrectomy induced angiogenesis in the injured kidney, which was evidenced by the up-regulation of the aforementioned molecules, whereas administration of pioglitazone significantly attenuated the elevation of the angiogenic markers both at mRNA and protein levels $(P<0.05)$. Moreover, administration of PPAR $\gamma$ inhibitor GW9662 blocked the anti-angiogenic effect of pioglitazone in renal failure, suggesting that activation of PPAR $\gamma$ pathway by pioglitazone may play a determinant role in the treatment of renal failure via inhibition of angiogenesis. 


\section{Discussion}

In the current study, the effect of pioglitazone on renal antioxidative capacity and angiogenesis in response to subtotal nephrectomy was investigated in a series of experiments. It was found that the agent was able to restore the structure and regular function of the injured kidney in the Nx rat model. This protective effect of pioglitazone was achieved by direct inhibition on the production of vascularization-related molecules, including NO, HIF$1 \alpha$, eNOS, VEGF, Flk-1 and Flt-1. Additionally, administration of pioglitazone also increased the expression of SOD while decreased the level of MDA, implying the restoration of antioxidative capacity in the injured kidney.

Pioglitazone is an agonist of PPAR $\gamma$ and is capable of lowering blood glucose level through enhancement of insulin resistance [28-30]. In the previous studies on the role of PPAR in diabetic kidney disease, it was found that the PPAR agonist could modulate systemic metabolism in a number of ways, including improvement of glycaemic control, insulin sensitivity and dyslipidaemia, and these modulatory effects might influence the outcome of the disease [31-34]. Later on, Zou et al. [21] and Reel et al. [35] concluded that pioglitazone was capable of protecting the kidney from renal injury by enhancing the antioxidative and anti-inflammatory capacity of the kidney. Furthermore, it was reported that pioglitazone significantly increased the expression of VEGF and accelerated the angiogenesis process following kidney injuries [22-24]. In the current study, the sclerosed areas and the levels of proteinuria, BUN and $\mathrm{Cr}$ in the Nx rat kidneys were markedly reduced after administration of pioglitazone, and these results substantiated the renoprotective effect of pioglitazone that was proposed in the previous studies. These results also evidently showed the potential of pioglitazone in the repair of structural and functional deterioration of the kidney in CKDs. Moreover, the antioxidative capacity of the injured kidney was also improved by pioglitazone. However, contrary to most previous studies, our data demonstrated that the angiogenesis in the injured kidney was inhibited, instead of being activated, by pioglitazone.

The results of ELISA, IHC, RT-qPCR, and western blotting showed that the expression of angiogenic factors, such as CD31, $\alpha$ SMA, HIF-1 $\alpha$, VEGF, eNOS, NO, Flt-1 and Flk-1, were upregulated in the injured kidney. In contrast, pioglitazone treatment on the Nx rats resulted in remarkable inhibition on the production of all these molecules. As the common markers of angiogenesis, the reduction of CD31 and $\alpha$ SMA evidently represented the inhibition of neovascularization. Although rarely reported, the repressive effect of pioglitazone on the expression of HIF-1 $\alpha$ and VEGF was demonstrated by Dromparis and colleagues [25]. Consistently, in the current study, pioglitazone-induced activation of PPAR $\gamma$ was capable of down-regulating HIF- $1 \alpha$ expression, probably through reduction of oxidative stress, resulting in inhibition of VEGF expression [36]. Subsequently, the levels of eNOS, an essential mediator of VEGF-induced angiogenesis, and its catalysate NO were both reduced as well [37]. Furthermore, the expressions of two distinct receptor tyrosine kinases, Flt-1 and Flk1, which were stimulated by VEGF, were suppressed due to down-regulation of VEGF [38]. However, the proposed regulatory pathway may not explain the pro-angiogenic effect of pioglitazone as demonstrated in the previous studies [22-24]. The inconsistent conclusions might result from different doses, various exposure time to pioglitazone, and even the method of model construction. Taken together, the outcome of pioglitazone treatment is sensitive to drug administration time and depends on the target organ. Hence, more comprehensive studies are needed to further elucidate the mechanism of pioglitazone-based therapies.

In conclusion, our study demonstrated that pioglitazone restored the structure and function of the injured kidneys in Nx rats. Administration of pioglitazone increased the antioxidative capacity while inhibited the expression of angiogenic factors in the injured kidney. Although the underlying mechanism involved in this treatment process is not fully understood, pharmacological modification of renal structure and function by pioglitazone might be valuable along with other cell transplantation or gene therapies for the treatment of CKDs.

\section{KARGER}




\section{Acknowledgements}

This study was supported by grants from the Social Development Project of Liaoning Province (No.: 2012225094), the Program for Liaoning Excellent Talents in University (No.: LJQ2013086), and the Science and Technology Project of Shenyang City (No.: F15-199-1-51).

\section{Disclosure Statement}

The authors declare no conflict of interest.

\section{References}

1 Fogo AB: Progression versus regression of chronic kidney disease. Nephrol Dial Transpl 2006;21:281-284.

2 Risdon RA, Sloper JC, De Wardener HE: Relationship between renal function and histological changes found in renal-biopsy specimens from patients with persistent glomerular nephritis. The Lancet 1968;292:363366.

3 Schainuck LI, Striker GE, Cutler RE, Benditt EP: Structural-functional correlations in renal disease. Part II. The correlations. Hum Pathol 1970;1:631-641.

4 Striker GE, Schainuck LI, Cutler RE, Benditt EP: Structural-functional correlations in renal disease. I. A method for assaying and classifying histopathologic changes in renal disease. Hum Pathol 1970;1:615-630.

5 Mackensen-Haen S, Bader R, Grund KE, Bohle A: Correlations between renal cortical interstitial fibrosis, atrophy of the proximal tubules and impairment of the glomerular filtration rate. Clin Nephrol 1981;15:167-171.

6 Nangaku M: Mechanisms of tubulointerstitial injury in the kidney: Final common pathways to end-stage renal failure. Intern Med 2004;43:9-17.

7 Eddy AA: Experimental insights into the tubulointerstitial disease accompanying primary glomerular lesions. J Am Soc Nephrol 1994;5:1273-1287.

8 Nath KA: Tubulointerstitial changes as a major determinant in the progression of renal damage. Am J Kidney Dis 1992;20:1-17.

9 Fine LG, Bandyopadhay D, Norman JT: Is there a common mechanism for the progression of different types of renal diseases other than proteinuria? Towards the unifying theme of chronic hypoxia. Kidney Int 2000;57:S22-S26.

10 Kang DH, Kanellis J, Hugo C, Truong L, Anderson S, Kerjaschki D, Schreiner GF, Johnson RJ: Role of the microvascular endothelium in progressive renal disease. J Am Soc Nephrol 2002;13:806-816.

11 Eckardt KU, Rosenberger C, Jurgensen JS, Wiesener MS: Role of hypoxia in the pathogenesis of renal disease. Blood purificat 2003;21:253-257.

12 Nangaku M: Hypoxia and tubulointerstitial injury: A final common pathway to end-stage renal failure. Nephron Exp Nephrol 2004;98:e8-12.

13 Evans RM: The steroid and thyroid hormone receptor superfamily. Science 1988;240:889-895.

14 Murphy GJ, Holder JC: Ppar- $\gamma$ agonists: Therapeutic role in diabetes, inflammation and cancer. Trends Pharmacol Sci 2000;21:469-474.

15 Spiegelman BM: Ppar-gamma: Adipogenic regulator and thiazolidinedione receptor. Diabetes 1998;47:507514.

16 Peraldi P, Xu M, Spiegelman BM: Thiazolidinediones block tumor necrosis factor-alpha-induced inhibition of insulin signaling. J Clinl Invest 1997;100:1863.

17 Hallakou S, Doare L, Foufelle F, Kergoat M, Guerre-Millo M, Berthault MF, Dugail I, Morin J, Auwerx J, Ferre P: Pioglitazone induces in vivo adipocyte differentiation in the obese zucker fa/fa rat. Diabetes 1997;46:1393-1399.

18 Qi HP, Wang Y, Zhang QH, Guo J, Li L, Cao YG, Li SZ, Li XL, Shi MM, Xu W: Activation of peroxisome

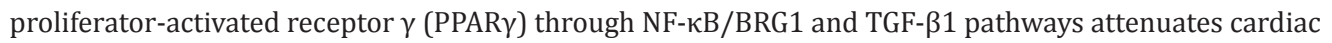
remodeling in pressure-overloaded rat hearts. Cell Physiol Biochem 2015;35:899-912. 


\section{Cellular Physiology Cell Physiol Biochem 2016;38:1831-1840

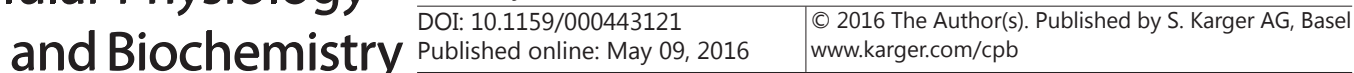

Sun et al.: Pioglitazone Antagonizes CKD via Augmentation of Antioxidative Capacity and Block of Angiogenesis

19 Wang S, Sun Z, Zhang X, Li Z, Wu M, Zhao W, Wang H, Chen T, Yan H, Zhu J: Wnt1 positively regulates cd36 expression via tcf4 and ppar-gamma in macrophages. Cell Physiol Biochem 2015;35:1289-1302.

20 Meng Y, Chen C, Tian C, Du J, Li HH: Angiotensin ii-induced egr-1 expression is suppressed by peroxisome proliferator-activated receptor- $\gamma$ ligand 15d-PGJ2 in macrophages. Cell Physiol Biochem 2015;35:689-698.

21 Zou C, Hu H, Xi X, Shi Z, Wang G, Huang X: Pioglitazone protects against renal ischemia-reperfusion injury by enhancing antioxidant capacity. J Surg Res 2013;184:1092-1095.

22 Huang PH, Sata M, Nishimatsu H, Sumi M, Hirata Y, Nagai R: Pioglitazone ameliorates endothelial dysfunction and restores ischemia-induced angiogenesis in diabetic mice. Biomed Pharmacother 2008;62:46-52.

23 Biscetti F, Straface G, Arena V, Stigliano E, Pecorini G, Rizzo P, De Angelis G, Iuliano L, Ghirlanda G, Flex A: Pioglitazone enhances collateral blood flow in ischemic hindlimb of diabetic mice through an aktdependent vegf-mediated mechanism, regardless of ppar $\gamma$ stimulation. Cardiovasc Diabetol 2009;8:49.

24 Kong X, Zhang DY, Wu HB, Li FX: Losartan and pioglitazone ameliorate nephropathy in experimental metabolic syndrome rats. Biol Pharm Bull 2011;34:693-699.

25 Dromparis P, Sutendra G, Paulin R, Proctor S, Michelakis ED, McMurtry MS: Pioglitazone inhibits hif1alpha-dependent angiogenesis in rats by paracrine and direct effects on endothelial cells. J Mol Med 2014;92:497-507.

26 Ghosh SS, Massey HD, Krieg R, Fazelbhoy ZA, Ghosh S, Sica DA, Fakhry I, Gehr TW: Curcumin ameliorates renal failure in 5/6 nephrectomized rats: Role of inflammation. Am J Physiol-Renal 2009;296:F1146-1157.

27 Ghosh S, Sica D, Schoolwerth AC, Quigg RJ, Haas M, Fakhry I, Gehr TW: The role of the renin-angiotensin system in cholesterol and puromycin mediated renal injury. Am J Med Sci 2002;324:296-304.

28 Gomes MB: Glitazones and the metabolic syndrome: Mechanism of action, pathophysiology and therapeutic indications. Arq Bras Endocrinol 2006;50:271-280.

29 Zhu C, Xiao Y, Liu X, Han J, Zhang J, Wei L, Jia W: Pioglitazone lowers serum retinol binding protein 4 by suppressing its expression in adipose tissue of obese rats. Cell Physiol Biochem 2015;35:778-788.

30 Chraibi A, Renauld S: Ppary-induced stimulation of amiloride-sensitive sodium current in renal collecting duct principal cells is serum and insulin dependent. Cell Physiol Biochem 2014;33:581-593.

31 Imano E, Kanda T, Nakatani Y, Nishida T, Arai K, Motomura M, Kajimoto Y, Yamasaki Y, Hori M: Effect of troglitazone on microalbuminuria in patients with incipient diabetic nephropathy. Diabetes Care 1998;21:2135-2139.

32 Nakamura T, Ushiyama C, Suzuki S, Shimada N, Sekizuka K, Ebihara L, Koide H: Effect of troglitazone on urinary albumin excretion and serum type iv collagen concentrations in type 2 diabetic patients with microalbuminuria or macroalbuminuria. Diabetic Med 2001;18:308-313.

33 Nakamura T, Ushiyama C, Shimada N, Hayashi K, Ebihara I, Koide H: Comparative effects of pioglitazone, glibenclamide, and voglibose on urinary endothelin-1 and albumin excretion in diabetes patients. J Diabetes Complicat 2000;14:250-254.

34 Nie H, Xue X, Li J, Liu X, Lv S, Guan G, Liu H, Liu G, Liu S, Chen Z: Nitro-oleic acid attenuates ogd/r-triggered apoptosis in renal tubular cells via inhibition of bax mitochondrial translocation in a ppar-gammadependent manner. Cell Physiol Biochem 2015;35:1201-1218.

35 Reel B, Guzeloglu M, Bagriyanik A, Atmaca S, Aykut K, Albayrak G, Hazan E: The effects of ppar-gamma agonist pioglitazone on renal ischemia/reperfusion injury in rats. J Surg Res 2013;182:176-184.

36 Lee KS, Kim SR, Park SJ, Park HS, Min KH, Jin SM, Lee MK, Kim UH, Lee YC: Peroxisome proliferator activated receptor- $\gamma$ modulates reactive oxygen species generation and activation of nuclear factor- $\kappa \mathrm{b}$ and hypoxia-inducible factor $1 \alpha$ in allergic airway disease of mice. J Allergy Clin Immun 2006;118:120-127.

37 Kroll J, Waltenberger J: Vegf-a induces expression of enos and inos in endothelial cells via vegf receptor-2 (kdr). Biochem Bioph Res Co 1998;252:743-746.

38 Waltenberger J, Claesson-Welsh L, Siegbahn A, Shibuya M, Heldin C-H: Different signal transduction properties of kdr and flt1, two receptors for vascular endothelial growth factor. J Biol Chem 1994;269:26988-26995. 